\title{
A surgery triangle for lattice cohomology
}

\author{
JOSHUA EVAN GREENE
}

\begin{abstract}
Lattice cohomology, defined by Némethi in [10], is an invariant of negative definite plumbed 3-manifolds which conjecturally computes their Heegaard Floer homology $\mathrm{HF}^{+}$. We prove a surgery exact triangle for the lattice cohomology analogous to the one for $\mathrm{HF}^{+}$. This is a step towards relating these two invariants.
\end{abstract}

57R58; 57M27, 53D40, 11H55

\section{Introduction}

\subsection{Context and previous work}

In this article, we study a model for the Heegaard Floer homology $\mathrm{HF}^{+}$of a negative definite plumbed manifold. Heegaard Floer homology is a powerful invariant in low-dimensional topology, and much research has gone into its algorithmic computability, from the seminal works by Sarkar and Wang [15] and Manolescu, Ozsváth and Sarkar [6] to the formidable paper by Manolescu and Ozsváth [5]. However, there lacks a transparent, efficient means of calculating the invariant in general, and negative definite plumbed manifolds are one family for which calculation seems feasible. Moreover, calculations in this case are useful in applications to knot concordance (see the author and Jabuka [4]), unknotting number (see Ozsváth and Szabó [14]) and smoothings of rational surface singularities (see Stipsicz [16]). In this setting, Némethi has given an elegant construction of an invariant called the lattice cohomology $\mathbb{H}^{+}$ which conjecturally computes $\mathrm{HF}^{+}$in [10], and this is the invariant we study here.

We first describe the family of manifolds at hand. Let $G=(V, E)$ denote a finite planar graph with an integer weight $m(v)$ for each vertex $v \in V$ and a sign \pm for each edge $e \in E$. Associated to $G$ is a compact $4-$ manifold with boundary $X(G)$. To describe it, center a round unknot at each vertex of $G$ with framing $m(v)$, and introduce a right-hand (resp. left-hand) clasp between neighboring unknots for each positive (resp. negative) edge $e \in E$. Let $\mathbb{L}$ denote the resulting framed link in $S^{3}=\partial D^{4}$, and let $X(G)$ denote the result of attaching 2-handles to $D^{4}$ along $\mathbb{L}$. When $G$ is acyclic (ie a disjoint union of trees), $X(G)$ admits an alternative description: form the disk bundle over $S^{2}$ with Euler number $m(v)$ for each $v \in V$, and plumb together the 
bundles of neighboring vertices for each edge $e \in E$. Note the signs on the edges of $G$ become immaterial in this case. We let $Y(G)$ denote the oriented boundary of $X(G)$.

The graph $G$ defines a free $\mathbb{Z}$-module $L=L(G)$ which is freely generated by classes $E_{v}, v \in V$. There is a symmetric bilinear form on this module which is defined by setting $\left(E_{v}, E_{w}\right)$ equal to the signed number of edges between $v$ and $w$, when $v \neq w$ and $\left(E_{v}, E_{v}\right)=m(v)$. The quadratic module $(L(G),(\cdot, \cdot))$ defined in this way is isomorphic to the module $H_{2}(X(G) ; \mathbb{Z})$ equipped with its intersection pairing. The isomorphism is set up by identifying a class $E_{v}$ with $\left[S_{v}\right]$, where $S_{v}$ is the sphere obtained by taking the union of a Seifert disk for $K_{v}$ with the core of the corresponding handle attachment. In the case of the plumbing description, $S_{v}$ may be alternately viewed as the base sphere in the disk bundle associated to $v \in V$.

Definition 1.1 A graph $G$ is negative definite if it is acyclic and the pairing $(\cdot, \cdot)$ is negative definite, and the resulting space $Y(G)$ is a negative definite plumbed manifold.

The spaces $X(G)$ associated to negative definite graphs are precisely the diffeomorphism types of good resolutions of normal surface singularities as by Grauert [2] and Mumford [7]; for a good summary, see Némethi [8, pages 282-283].

Ozsváth and Szabó undertook the determination of $\mathrm{HF}^{+}$of a negative definite plumbed manifold under an additional assumption on the graph $G$ [13]. Namely, they call a vertex $v \in V$ bad if $m(v)+d(v)>0$; here $d(v)$ denotes the number of edges incident $v$. Their result is a calculable description of the invariant $\mathrm{HF}^{+}(-Y(G))$ under the assumption that $G$ has at most one bad vertex. Additionally, they describe the "even half" of this invariant $H F_{\text {even }}^{+}(-Y(G))$ when $G$ has two bad vertices. Using their result, Ozsváth and Szabó calculated the invariant for many spaces of interest, and gave a useful formula for the correction terms of such a space.

However, it remained a problem to find a suitable generalization of their algorithm which would apply to any negative definite plumbed 3-manifold $Y$. This was taken up by Némethi, who proposed the lattice cohomology $\mathbb{H}^{+}(Y)$. He proved that the lattice cohomology is a diffeomorphism invariant of $Y$ (independent of the negative definite graph used to present it), reduces to Ozsváth's and Szabó's model in the domain for which they defined it, and also agrees with $\mathrm{HF}^{+}$for the boundaries of rational and elliptic surface singularities $[9 ; 10]$. As a result, Némethi was able to show that rational surface singularities are $L$-spaces; and his conjecture $\mathbb{H}^{+}(Y) \cong \mathrm{HF}^{+}(Y)$ would imply that these are all the $L$-spaces amongst negative definite plumbed manifolds. At present there is no purely topological characterization of $L$-spaces, and this remains a fascinating problem. 


\subsection{The main result}

A key feature of the Floer homology groups is that they obey a surgery exact triangle: if $K \subset Y$ is a framed knot, then there is a long exact sequence

$$
\cdots \rightarrow H F^{+}(Y) \rightarrow H F^{+}\left(Y_{0}(K)\right) \rightarrow H F^{+}\left(Y_{1}(K)\right) \rightarrow H F^{+}(Y) \rightarrow \cdots ;
$$

here $Y_{r}(K)$ denotes the result of $r$-framed surgery on $K$. As a first step towards proving Némethi's conjecture, it is desirable to know that the lattice cohomology obeys an analogous exact triangle.

The purpose of this article is to prove Theorem 3.1, which establishes the existence of the surgery exact triangle for the lattice cohomology over the coefficient ring $\mathbb{Z} / 2 \mathbb{Z}$. Moreover, we describe a version of the lattice cohomology which applies to the family of manifolds $Y(G)$ without any additional assumption on the graph $G$, and show that the triangle holds in this setting. Indeed, the proof of the triangle is a matter of fairly straightforward (if somewhat involved) algebra once we have suitably defined the invariant $\mathbb{H}^{+}$for arbitrary $Y(G)$.

Finally, and what is perhaps most intriguing, the lattice cohomology comes equipped with an additional integer grading which has no obvious counterpart in the Floer homology. If $L \subset S^{3}$ is a link, then the double cover of $S^{3}$ branched along $L$ takes the form $Y(G)$ for some $G$, and conversely all negative definite plumbed manifolds arise in this way. For branched double-covers there is a conjectural $\delta$-grading described by Baldwin in [1] and by the author in [3, Conjecture 8.1]. Indeed, these two quantities agree (up to an overall shift) in the limited domain where they have both been computed. In light of the way we expect the $\delta$-grading to arise from [1], it is compelling to search for a more direct relationship between Khovanov homology and lattice cohomology.

\subsection{Update}

Since this article first appeared as a preprint, Némethi reproved Theorem 3.1, found the sign assignment required to establish it over $\mathbb{Z}$, and established a related exact triangle which does not mix $\operatorname{spin}^{c}$ structures [11]. Ozsváth, Stipsicz and Szabó also reproved Theorem 3.1 and used it to establish the existence of a spectral sequence $\mathbb{H}^{+} \rightsquigarrow H F^{+}$, which is a further step towards establishing the conjectured isomorphism between them [12].

Acknowledgments Thanks to my advisor Zoltán Szabó for encouraging me to pursue this project and to András Némethi for helpful correspondence. 


\section{The lattice cohomology}

The pairing on $L(G)$ gives rise to a quadratic form $q(x)=-\frac{1}{8}(x, x)$, and extends to a pairing on $L \otimes \mathbb{R}$. We say that a vector $K \in \operatorname{Hom}(L, \mathbb{Z})$ is characteristic if $(K, x) \equiv(x, x)(\bmod 2)$ for all $x \in L(G)$; and the set of all characteristic vectors is denoted $\operatorname{Char}(G)$. Let

$$
\mathcal{C}^{+}(G) \subset \operatorname{Hom}_{\mathbb{F}}\left(\operatorname{Char}(G) \times \mathcal{P}(V), \mathcal{T}_{0}^{+}\right)
$$

consist of the maps with finite support. Here $\mathbb{F}$ denotes either $\mathbb{Z}$ or $\mathbb{Z} / 2 \mathbb{Z} ; \mathcal{P}(V)$, the power set of $V$; and $\mathcal{T}_{0}^{+}$, the $\mathbb{F}[U]$-module $\mathbb{F}\left[U, U^{-1}\right] / U \cdot \mathbb{F}[U]$. The latter is a graded $\mathbb{F}$-module, with grading given by $\operatorname{gr}\left(U^{-d}\right)=2 d$, and makes $\mathcal{C}^{+}(G)$ into an $\mathbb{F}[U]$-module. The group $\mathcal{C}^{+}(G)$ has an additional grading, where $\phi \in \mathcal{C}^{+}(G)$ is homogeneous of degree $s$ if it is supported on pairs $(K, S)$ with $|S|=s$.

Consider the affine lattice $K+2 L \subset L \otimes \mathbb{R}$. Associated to a pair $\left(K^{\prime}, S\right) \in(K+2 L) \times \mathcal{P}(V)$ is an $s$-dimensional cube in $L \otimes \mathbb{R}$ with vertex set $\left\{K^{\prime}+2 \sum_{j \in T} E_{j} \mid T \subset S\right\}$. In this way we obtain a cubical decomposition of $L \otimes \mathbb{R}$, and in turn a chain complex $\left(C_{S}, \partial\right)$. Under the identification between pairs $(K, S)$ and cubes, we can express the boundary operator as

$$
\partial(K, S)=\sum_{\left(K^{\prime}, S^{\prime}\right)} \epsilon\left(K^{\prime}, S^{\prime}\right) \cdot\left(K^{\prime}, S^{\prime}\right),
$$

where $\epsilon\left(K^{\prime}, S^{\prime}\right)= \pm 1$ for pairs of the form $(K, S-w)$ or $\left(K+2 E_{w}, S-w\right), w \in S$, and $\epsilon\left(K^{\prime}, S^{\prime}\right)=0$ otherwise. Lastly, we extend the quadratic form $q$ to a function on $\operatorname{Char}(G) \times \mathcal{P}(V)$ by setting

$$
q(K, S)=\max \left\{q\left(K+2 \sum_{j \in T} E_{j}\right) \mid T \subset S\right\} .
$$

We define a differential $\delta$ on the group $\mathcal{C}^{+}(G)$ by setting

$$
\delta(\phi)(K, S)=\sum_{\left(K^{\prime}, S^{\prime}\right)} \epsilon\left(K^{\prime}, S^{\prime}\right) \cdot U^{q(K, S)-q\left(K^{\prime}, S^{\prime}\right)} \cdot \phi\left(K^{\prime}, S^{\prime}\right) .
$$

It is straightforward to verify $\delta^{2}=0$, making use of the fact $\partial^{2}=0$ [10, Lemma 3.1.5]. The following definition is in essence [10, Definition 3.2.5].

Definition 2.1 The homology of $\mathcal{C}^{+}(G)$, regarded as a graded $\mathbb{F}[U]$-module, is the lattice cohomology of $G$. It is denoted by $\mathbb{H}^{+}(G)$.

Observe that this definition makes sense for any finite graph $G$, with no assumption on planarity; however, it is unclear what significance it has in this more general setting. 
In the case that $G$ is negative definite, there is a more topological description of the invariant $\mathbb{H}^{+}(G)$ [10, Definition 3.1.11], though we do not use it here. This is the invariant we wish to compare to $\mathrm{HF}^{+}(-Y(G))$. In fact, [10, Conjecture 5.2.4] proposes in a precise form the conjecture that $\mathbb{H}^{+}(G) \cong H F^{+}(-Y(G))$ in the case that $G$ is negative definite.

\section{Statement of the triangle}

The invariant $\mathrm{HF}^{+}$obeys an exact triangle. For the case of interest, this triangle takes the following form. Set $Y=Y(G)$, select $v \in V$, and let $K$ denote a meridian for the link component $K_{v}$. The knot $K$ inherits a natural framing when viewed as an unknotted curve in $S^{3}$. Then $Y_{0}(K) \cong Y(G-v)$ and $Y_{-1}(K) \cong Y\left(G_{+1}(v)\right)$, where $G-v$ denotes the graph gotten by deleting $v$ and all its incident edges, and $G_{+1}(v)$ denotes the graph gotten by increasing $m(v)$ by one. Thus the triangle reads

$$
\begin{aligned}
\cdots \rightarrow H F^{+}\left(-Y\left(G_{+1}(v)\right)\right) \rightarrow H F^{+}(-Y(G)) \rightarrow H F^{+} & (-Y(G-v)) \\
& \rightarrow H F^{+}\left(-Y\left(G_{+1}(v)\right)\right) \rightarrow \cdots .
\end{aligned}
$$

Our purpose here is to prove an analogous result for the lattice cohomology.

Theorem 3.1 Let $\mathbb{F}$ denote the coefficient ring $\mathbb{Z} / 2 \mathbb{Z}$. There is a short exact sequence of complexes

$$
0 \rightarrow \mathcal{C}^{+}\left(G_{+1}(v)\right) \stackrel{A}{\longrightarrow} \mathcal{C}^{+}(G) \stackrel{B}{\longrightarrow} \mathcal{C}^{+}(G-v) \rightarrow 0,
$$

which gives rise to an $\mathbb{F}[U]$-equivariant exact triangle of lattice cohomology groups

$$
\cdots \rightarrow \mathbb{H}^{+}\left(G_{+1}(v)\right) \rightarrow \mathbb{H}^{+}(G) \rightarrow \mathbb{H}^{+}(G-v) \rightarrow \mathbb{H}^{+}\left(G_{+1}(v)\right) \rightarrow \cdots
$$

The rest of this article is devoted to the proof of Theorem 3.1, which we break into several pieces.

\section{Definition of maps appearing in the short exact sequence}

There is a canonical identification between $L(G-v)$ and the sublattices of $L(G)$ and $L\left(G_{+1}(v)\right)$ spanned by the classes $E_{w}, w \neq v$. We denote a characteristic vector for $G$ or $G_{+1}(v)$ by a pair $(K, t)$, where $K$ denotes the restriction to $L(G-v)$ and $t$ is the pairing of the vector with $E_{v}$. Denote by $(\cdot, \cdot)^{\prime}$ the pairing on $L\left(G_{+1}(v)\right)$ and by $q^{\prime}$ the associated function on $\operatorname{Char}\left(G_{+1}(v)\right) \times \mathcal{P}(V)$. 
The definition of the maps $A$ and $B$ on the 0 -th level of the short exact sequence is taken from [13]. We set

$$
\begin{gathered}
A(\phi)(K, t)=\sum_{i=-\infty}^{+\infty} U^{i(i+1) / 2} \cdot \phi(K, t+2 i+1), \\
B(\phi)(K)=\sum_{i=-\infty}^{+\infty} \phi(K, m(v)+2 i) .
\end{gathered}
$$

(We suppress $S=\varnothing$ from the notation.) Both sums are finite, since $\phi$ has finite support. Observe that $A=\mathbb{A}^{+} \circ R$ and $B=\mathbb{B}^{+}$, where the maps $\mathbb{A}^{+}$and $\mathbb{B}^{+}$appear in [13, Lemma 2.9, Proposition 2.5].

Let us attempt to extend this definition to a pair of $\mathbb{F}[U]$-equivariant chain maps $A: \mathcal{C}^{+}\left(G_{+1}(v)\right) \rightarrow \mathcal{C}^{+}(G)$ and $B: \mathcal{C}^{+}(G) \rightarrow \mathcal{C}^{+}(G-v)$. We focus on the definition of $A$ first. The condition $\delta A=A \delta$ and a straightforward induction on $|S|$ show that the value of $A(\phi)((K, t), S)$ is an $\mathbb{F}[U]$-linear combination of terms $\phi((K, t+2 i+1), S)$. Moreover, the coefficient on $\phi((K, t+2 i+1), S)$ is a monomial $U^{c(i,(K, t), S)}$, for some $c(i,(K, t), S) \geq 0$. We determine the value of this exponent by comparing the coefficients on $\phi((K, t+2 i+1), S-w)$ on both sides of the identity $\delta A(\phi)((K, t), S)=A \delta(\phi)((K, t), S)$, for $w \in S$. An induction on $|S|$ shows that this value is uniquely determined by the expression

$$
\begin{aligned}
c(i,(K, t), S)= & {[q((K, t), S)-q((K, t))] } \\
& -\left[q^{\prime}((K, t+2 i+1), S)-q^{\prime}(K, t+2 i+1)\right]+i(i+1) / 2 .
\end{aligned}
$$

However, both sides of the identity $\delta A(\phi)((K, t), S)=A \delta(\phi)((K, t), S)$ also involve terms of the form $\phi\left((K, t+2 i+1)+2 E_{w}, S-w\right)$. It stands to check that the stated definition of $c(i,(K, t), S)$ makes the coefficients on these terms agree as well. This is easily confirmed for the case when $w \neq v$. In the case $w=v$, there is one small wrinkle. Namely, we must be careful to recognize the class $(K, t+2 i-1)+2 E_{v} \in \operatorname{Char}\left(G_{+1}(v)\right)$ is the one whose evaluation on $E_{w}$ with respect to the pairing $(\cdot, \cdot)^{\prime}$ is given by $\left(K+2 E_{v}, E_{w}\right)$ when $w \neq v$ and $\left(K+2 E_{v}, E_{v}\right)+2 i+1$ when $w=v$. This is due to the fact that $\left(E_{v}, E_{v}\right)^{\prime}=\left(E_{v}, E_{v}\right)+1$. Having observed this, the case $w=v$ follows as well.

Next, we need to verify that $c(i,(K, t), S) \geq 0$, so that $A$ is a bona fide $\mathbb{F}[U]$-module map, rather than just an $\mathbb{F}\left[U, U^{-1}\right]$-module map. The value of $q((K, t), S)-q(K, t)$ is the maximum of the values $q\left((K, t)+2 E_{T}\right)-q(K, t)$, where $E_{T}:=\sum_{j \in T} E_{j}, T \subset S$. 
It is easy to check that

$q^{\prime}\left((K, t+2 i+1)+2 E_{T}\right)-q^{\prime}(K, t+2 i+1)= \begin{cases}q\left((K, t)+2 E_{T}\right)-q(K, t), & v \notin T, \\ q\left((K, t)+2 E_{T}\right)-q(K, t)-i-1, & v \in T .\end{cases}$

It follows that if $v \notin S$, then

$$
q^{\prime}((K, t+2 i+1), S)=q(K, S),
$$

and if $v \in S$, then

$$
q^{\prime}((K, t+2 i+1), S)=\max \left\{q((K, t), S-v), q\left((K, t)+2 E_{v}, S-v\right)-i-1\right\} .
$$

Denote by $r((K, t), S)$ the difference $q((K, t), S-v)-q\left((K, t)+2 E_{v}, S-v\right)$ when $v \in S$. We conclude that if $v \notin S$, then

$$
c(i,(K, t), S)=i(i+1) / 2
$$

and if $v \in S$, then

(5) $c(i,(K, t), S)= \begin{cases}i(i+1) / 2, & r((K, t), S) \geq \max \{0, i+1\}, \\ (i+1)(i+2) / 2, & r((K, t), S) \leq \min \{0, i+1\}, \\ i(i+1) / 2+r(K, S), & 0 \leq r((K, t), S) \leq i+1, \\ (i+1)(i+2) / 2-r(K, S), & i+1 \leq r((K, t), S) \leq 0 .\end{cases}$

Observe that in any case, $c(i,(K, t), S) \geq 0$, so that $A$ is indeed an $\mathbb{F}[U]$-module map. Moreover, we see that for all $((K, t), S)$, the value $c(i,(K, t), S)$ is 0 for $i=-1$ and positive for $i>-1$ and $i<-2$.

The extension of $B$ to a chain map $\mathcal{C}^{+}(G) \rightarrow \mathcal{C}^{+}(G-v)$ proceeds similarly, but is simpler. The result is summarized as follows.

Proposition 4.1 There is a unique way to extend the maps $A$ and $B$ in Equations (1) and (2) to $\mathbb{F}[U]$-equivariant chain maps $A: \mathcal{C}^{+}\left(G_{+1}(v)\right) \rightarrow \mathcal{C}^{+}(G)$ and $B: \mathcal{C}^{+}(G) \rightarrow \mathcal{C}^{+}(G-v)$. They are defined by setting

$$
A(\phi)((K, t), S)=\sum_{i=-\infty}^{+\infty} U^{c(i,(K, t), S)} \cdot \phi((K, t+2 i+1), S)
$$

where $c(i,(K, t), S)$ is defined by Equation (3), and

$$
B(\phi)((K, t), S)=\sum_{i=-\infty}^{+\infty} \phi((K, m(v)+2 i+1), S) .
$$




\section{Proof of exactness}

\section{1 $A$ is injective}

Select a nonzero element $\phi \in \mathcal{C}^{+}(G)$. There is a filtration $\mathcal{F}$ on $\mathcal{T}_{0}^{+}$induced by the grading $\operatorname{gr}\left(U^{-d}\right)=2 d$, and where by convention $\mathcal{F}(0)=-\infty$. Choose a pair $((K, t), S)$ such that

(a) $\mathcal{F}(\phi((K, t), S))$ is maximal,

(b) $t$ is minimal subject to condition (a).

We have

$$
\mathcal{F}(A(\phi)((K, t-1), S))=\mathcal{F}\left(\sum_{i=-\infty}^{+\infty} U^{c(i,(K, t-1), S)} \cdot \phi((K, t+2 i), S)\right) .
$$

By condition (a) and the remarks following Equation (5), the $i=-1$ term in the sum has larger filtration grading than all the terms with $i>-1$; by condition (b), it has larger filtration grading than all those with $i<-1$ as well. Therefore, the value of $\mathcal{F}(A(\phi)((K, t-1), S))$ simply reduces to $\mathcal{F}(\phi((K, t), S)$, and this is nonzero, since $\phi$ is. It follows that $A(\phi) \neq 0$, so $A$ is injective.

\section{$5.2 B$ is surjective}

Denote by $(K, S)^{\vee}$ the Hom-dual to the pair $(K, S)$. Thus $\mathcal{C}^{+}(G-v)$ is freely generated as an $\mathbb{F}$-module by the maps $U^{-m} \cdot(K, S)^{\vee}, m \geq 0$. It is clear that

$$
B\left(U^{-m} \cdot((K, t), S)^{\vee}\right)=U^{-m} \cdot(K, S)^{\vee}
$$

for any $(K, S)$ and $t \equiv m(v)(\bmod 2)$. Hence $B$ is surjective.

\section{$5.3 \operatorname{im} A \subset \operatorname{ker} B$}

We compute

$$
\begin{aligned}
B \circ A(\phi)(K, S) & =\sum_{i=-\infty}^{+\infty} \sum_{j=-\infty}^{+\infty} U^{c(j,(K, m(v)+2 i+1), S)} \cdot \phi((K, m(v)+2 i+2 j+1), S) \\
& =\sum_{k=-\infty}^{+\infty} \sum_{j=-\infty}^{+\infty} U^{c(j,(K, m(v)+2(k-j)+1), S)} \cdot \phi((K, m(v)+2 k+1), S) .
\end{aligned}
$$

Since $S \subset V(G-v)$, we have by Equation (4) that $c(j,(K, m(v)+2(k-j)+1), S)=$ $j(j+1) / 2$ for all $k$ and $j$. For fixed $k$, the multiplier $U^{j(j+1) / 2}$ annihilates $\phi((K, m(v)+2 k+1), S)$ for $|j| \gg 0$. Those which do not annihilate this term cancel in pairs since $j(j+1) / 2$ is symmetric in $j$ and $-1-j$. Hence $B \circ A(\phi)=0$. 


\section{$5.4 \operatorname{ker} B=\mathcal{D}$}

We define $\mathcal{D}_{1} \subset \mathcal{C}^{+}(G)$ to be the submodule freely generated over $\mathbb{F}$ by the mappings

$$
U^{-m} \cdot((K, t), S)^{\vee}, \quad v \in S,
$$

$\mathcal{D}_{2} \subset \mathcal{C}^{+}(G)$ to be the submodule freely generated over $\mathbb{F}$ by

$$
U^{-m} \cdot\left[((K, t), S)^{\vee}+((K, t+2), S)^{\vee}\right], \quad v \notin S,
$$

and $\mathcal{D}=\mathcal{D}_{1} \oplus \mathcal{D}_{2}$. It is easy to see that $\phi \in \mathcal{D}$ if and only if in the expansion of $\phi$ with respect to the basis $\left\{U^{-m} \cdot((K, t), S)^{\vee}\right\}$ of $\mathcal{C}^{+}(G)$, there are an even number of terms $U^{-m} \cdot((K, t+2 i), S)^{\vee}$ as $i$ varies and $m,(K, t)$ and $v \notin S$ remain fixed. On the other hand, $\operatorname{ker} B$ clearly fulfills this description as well. Hence $\operatorname{ker} B=\mathcal{D}$.

\section{$5.5 \mathcal{D} \subset \operatorname{im} A$}

Filter $\mathcal{C}^{+}(G)$ by letting $\mathcal{C}^{(m)}$ consist of those maps whose image lies in the $m$-th filtered piece of $\mathcal{T}_{0}^{+}$, and set $\mathcal{D}_{1}^{(m)}=\mathcal{D}_{1} \cap \mathcal{C}^{(m)}, \mathcal{D}_{2}^{(m)}=\mathcal{D}_{2} \cap \mathcal{C}^{(m)}$, and $\mathcal{D}^{(m)}=\mathcal{D} \cap \mathcal{C}^{(m)}$. We prove by induction on $m$ that $\mathcal{D}^{(m)} \subset \operatorname{im} A$.

Fix a pair $((K, t), S)$ with $v \notin S$, and consider the map $((K, t+1), S)^{\vee} \in \mathcal{C}^{+}\left(G_{+1}(v)\right)$. Observe that in this case Equation (4) computes the value of $c(i,(K, t), S)$. From this it is clear that $A\left(((K, t+1), S)^{\vee}\right)$ vanishes on all pairs $\left(\left(K^{\prime}, t^{\prime}\right), S^{\prime}\right)$ not of the form $((K, t+2 i), S)$, and for this pair it takes the value 1 if $i=0$ or 1 , and 0 otherwise. In other words, $A\left(((K, t+1), S)^{\vee}\right)=((K, t), S)^{\vee}+((K, t+2), S)^{\vee}$. Hence $\mathcal{D}_{2}^{(0)} \subset \operatorname{im} A$.

Next, fix a pair $((K, t), S)$ with $v \in S$, and consider the map $((K, t+2 i+1), S)^{\vee} \in$ $\mathcal{C}^{+}\left(G_{+1}(v)\right)$. Once again, the image of this map under $A$ will vanish on all pairs not of the form $((K, t+2 j), S)$, and for a pair of this form it takes the value $U^{c(i-j,(K, t+2 j), S)}$. This value vanishes unless $c(i-j,(K, t+2 j), S)=0$, and we can determine when this occurs by way of Equation (5). First, observe that $r((K, t+2 j), S)=r(K, S)+j$ (recall the definition just before Equation (4)). Therefore, in working with the family of pairs $((K, t+2 j), S)$ as $j$ varies, we may assume that $K$ is chosen so that $r((K, t), S)=0$. With this choice made, Equation (5) implies that $c(i-j,(K, t+2 j), S)=0$ if and only if (a) $j=i \geq 0$, (b) $j=i+1$, or (c) $j=i+2, i \leq-2$. It follows that

$$
A\left(((K, t+2 i+1), S)^{\vee}\right)= \begin{cases}((K, t+2 i), S)^{\vee}+((K, t+2 i+2), S)^{\vee} & i \geq 0, \\ ((K, t), S)^{\vee} & i=-1, \\ ((K, t+2 i+2), S)^{\vee}+((K, t+2 i+4), S)^{\vee} & i \leq-2 .\end{cases}
$$


By taking linear combinations of these terms, we see at once $((K, t+2 i), S)^{\vee} \in \operatorname{im} A$ for all $i$. Hence $\mathcal{D}_{1}^{(0)} \subset \operatorname{im} A$, and so $\mathcal{D}^{(0)} \subset \operatorname{im} A$.

Now suppose that we have shown that $\mathcal{D}^{(m-1)} \subset \operatorname{im} A$ for some $m \geq 1$, and choose $\phi \in \mathcal{D}^{(m)}-\mathcal{D}^{(m-1)}$. Then $U \cdot \phi \in \mathcal{D}^{(m-1)} \subset \operatorname{im} A$ : say $A(\psi)=U \cdot \phi$. Then $A\left(U^{-1} \cdot \psi\right)=\phi+\rho$ for some $\rho \in \mathcal{C}^{(0)}$. Since im $A \subset \mathcal{D}$ by Sections 5.3 and 5.4, we have $A\left(U^{-1} \cdot \psi\right) \in \mathcal{D}$, and so $\rho=A\left(U^{-1} \cdot \psi\right)-\phi \in \mathcal{D}^{(0)}$. On the other hand, we have just seen that $\mathcal{D}^{(0)} \subset \operatorname{im} A$. Hence $\phi=A\left(U^{-1} \cdot \psi\right)-\rho \in \operatorname{im} A$, completing the induction step. In conclusion, $\mathcal{D} \subset \operatorname{im} A$.

Collecting the results of the previous subsections, we conclude that the chain maps $A$ and $B$ appearing in Proposition 4.1 provide the desired maps for the short exact sequence in Theorem 3.1. The resulting exact triangle of lattice cohomology groups follows directly.

\section{References}

[1] J A Baldwin, On the spectral sequence from Khovanov homology to Heegaard Floer homology, Int. Math. Res. Not. 2011 (2011) 3426-3470 MR2822178

[2] H Grauert, Über Modifikationen und exzeptionelle analytische Mengen, Math. Ann. 146 (1962) 331-368 MR0137127

[3] J E Greene, A spanning tree model for the Heegaard Floer homology of a branched double-cover, J. Topol., to appear

[4] J E Greene, S Jabuka, The slice-ribbon conjecture for 3-stranded pretzel knots, Amer. J. Math. 133 (2011) 555-580 MR2808326

[5] C Manolescu, P Ozsváth, Heegaard Floer homology and integer surgeries on links arXiv: 1011.1317

[6] C Manolescu, P Ozsváth, S Sarkar, A combinatorial description of knot Floer homology, Ann. of Math. 169 (2009) 633-660 MR2480614

[7] D Mumford, The topology of normal singularities of an algebraic surface and a criterion for simplicity, Inst. Hautes Études Sci. Publ. Math. (1961) 5-22 MR0153682

[8] A Némethi, Five lectures on normal surface singularities, from: "Low dimensional topology”, (K Böröczky, Jr, W Neumann, A Stipsicz, editors), Bolyai Soc. Math. Stud. 8, János Bolyai Math. Soc., Budapest (1999) 269-351 MR1747271

[9] A Némethi, On the Ozsváth-Szabó invariant of negative definite plumbed 3-manifolds, Geom. Topol. 9 (2005) 991-1042 MR2140997

[10] A Némethi, Lattice cohomology of normal surface singularities, Publ. Res. Inst. Math. Sci. 44 (2008) 507-543 
[11] A Némethi, Two exact sequences for lattice cohomology, from: "Noncommutative geometry and global analysis", (A Connes, A Gorokhovsky, M Lesch, M Pflaum, B Rangipour, editors), Contemp. Math. 546, Amer. Math. Soc., Providence, RI (2011) 249-269 MR2815139

[12] P Ozsváth, A Stipsicz, Z Szabó, A spectral sequence on lattice homology arXiv: $1206: 1654$

[13] P Ozsváth, Z Szabó, On the Floer homology of plumbed three-manifolds, Geom. Topol. 7 (2003) 185-224 MR1988284

[14] P Ozsváth, Z Szabó, Knots with unknotting number one and Heegaard Floer homology, Topology 44 (2005) 705-745 MR2136532

[15] S Sarkar, J Wang, An algorithm for computing some Heegaard Floer homologies, Ann. of Math. 171 (2010) 1213-1236

[16] A I Stipsicz, On the $\bar{\mu}$-invariant of rational surface singularities, Proc. Amer. Math. Soc. 136 (2008) 3815-3823 MR2425720

Department of Mathematics, Boston College

Carney Hall, Chestnut Hill, MA 02467, USA

joshua.greene@bc.edu

Received: 12 June 2012 